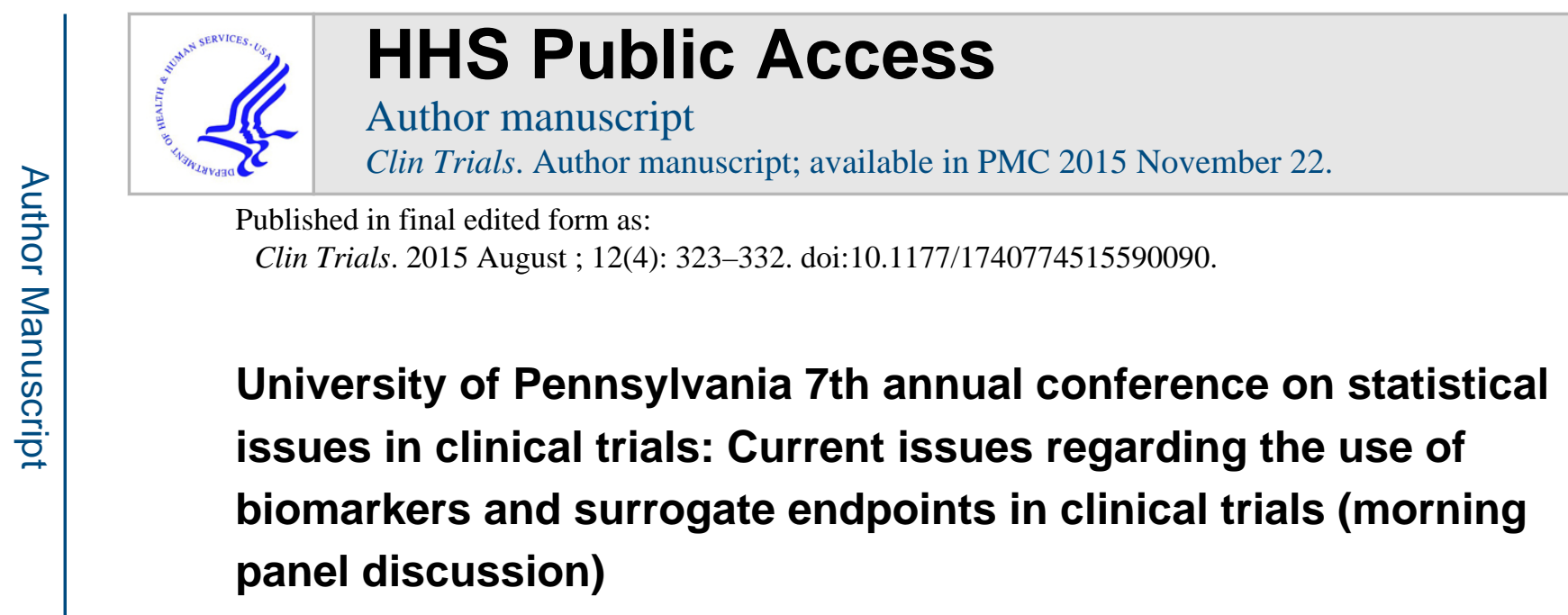

Michael Daniels, Constantine Frangakis, Vivek Charu, and Debashis Ghosh

\title{
Michael Daniels
}

Dr Taylor ${ }^{1}$ talked about a set of potential outcomes that he dealt with using what I will just call a highly structured parametric model. He considered multivariate normality when the outcomes and the surrogates are continuous or normal and otherwise using a Gaussian copula, which gives you a lot more flexibility on the margins, but has a similar underlying dependence structure. The really attractive thing about that sort of framework is there are four unidentified parameters and their correlations, which on the surface might seem problematic, but they live on a nice bounded space. So you can use default priors that are proper and you can make proper inferences accounting for the uncertainty about those parameters. In addition, as Dr Taylor noted, you can very easily put restrictions on them, for example, constrain them to be positive. But we need to remember that the whole framework relies on a parametric model. So, to use this sort of framework, it is extremely important to do careful model checking on how this multivariate normal fits the observed data. The fit is just related to two bivariate normals, the outcome and marker under the intervention (T1, $\mathrm{S} 1)$ and the outcome and the marker under the control (T0, S0) and that's all that you can check; the other assumptions about the joint of (T1, S1, T0, S0) are uncheckable (from the observed data), but needed to identify the causal effects. Another approach to these sorts of causal models, as opposed to thinking about a fully parametric model for all the potential outcomes, with some parameters that cannot be identified from the observed data, is to think about breaking it into two pieces. The data can tell you about some parts of the model, that

\section{Participants}

Michael Daniels, ScD, University of Texas at Austin

Constantine Frangakis, PhD, Johns Hopkins University

Vivek Charu, Johns Hopkins University

Debashis Ghosh, PhD, University of Colorado

William L Mietlowski, PhD, Novartis Oncology

Elizabeth Kumm, MS, MA, I3Statprobe

Marshall Joffe, MD, MPH, PhD, University of Pennsylvania

Jeremy Taylor, PhD, University of Michigan

Songbai Wang, Janssen Diagnostics

Stuart Baker, ScD, National Cancer Institute

Herb Weisberg, PhD, Causalytics, LLC

Chen Hu, PhD, American College of Radiology

Susan Ellenberg, PhD, University of Pennsylvania

Declaration of conflicting interests

Dr Herbert Weisberg reported that he holds US patent no. 8,688,610 B1, granted 1 April 2014, for the Cadit regression approach presented at the conference. No other conflicts of interest reported. 
is, the model for the observed data; then you make assumptions to identify whatever causal quantities you are interested in. With this approach, you can be very flexible with modeling the observed data because for that observed data you can just use Bayesian nonparametrics. Most of the Bayesian nonparametric models are grounded in some sort of parametric model, but they allow some robustness to parametric assumptions. You can think about this approach as similar in spirit to semiparametric approaches with estimating equations where you don't want to make parametric assumptions. You can do a similar sort of thing here and then have a (potentially) different set of assumptions to identify the causal parameters. Implicitly within a parametric model, you know the form of, for example, the conditional distributions between $\mathrm{T} 1$ and S0, and then there is an unidentified parameter that you have to deal with. We have a similar situation here of equating an unidentified conditional distribution to an identified one with an exponential tilt sensitivity parameter, lambda. You can get a slightly more expansive sensitivity analysis here, but it is going to be harder because this lambda parameter doesn't live on a bounded space like the correlations do. So you are going to have to be much more careful in terms of how you construct an informative prior on this sort of space. In general, Bayesian inference is extremely powerful in dealing with these causal settings because you are making assumptions that you can't check. We saw that from basically all the talks this morning. The Bayesian paradigm with priors allows you to characterize some uncertainty associated with the unknowns, as opposed to implicitly having point masses. It's important to think about how to frame these problems in the Bayesian setting; you can think about using a parametric model or the two-piece approach that I described. The framework I am talking about is not within principal stratification, so there are the direct and indirect effects that Dr Joffe talked about that fall out of that. We have some work related to this issue in the context of mediation, but it is basically the surrogate problem as well.

Dr Baker ${ }^{2}$ addressed causal association and a meta-analytic framework and had three parts to his talk. The first part dealt with extrapolation. Essentially, you want to predict the treatment effect on the outcome given the surrogate and you have information from historical data on that conditional distribution and then you are basically saying that the conditional distribution is going to be the same in the new trial. You are not saying anything about the marginal distribution of the surrogate. The assumption about the extrapolation is not checkable. It is an extrapolation, and it is connected to missing data problems because you have to deal with a similar unidentified distribution if you are analyzing missing data. There is also similar identification. What it looks like is a "missing at random" type of assumption; in the missing data literature, when you factor the joint distribution of missingness and the outcomes, the corresponding piece is often called the extrapolation distribution because it corresponds to an extrapolation. This raises the question of whether we should be accounting for uncertainty about such an assumption when we are applying meta-analytic approaches to surrogate marker assessments. Should we do some sensitivity checking of this assumption? Should we account for extra uncertainty about it because it is uncheckable? How might you do that? Dr Baker this morning, and Ellenberg ${ }^{3}$ in a previous article, talked about three components that you need to use this sort of approach: similar biological mechanisms, similar effect of patient managements after endpoint, and no harmful side effects. To some extent, based on the reliability of these assumptions, you can 
think about how that might introduce potential bias, in terms of a shift in the slope, if you want to think about it in terms of a linear relationship. You might add a little bit of uncertainty associated with the shift in the slope (via a prior). It seems like to fairly assess whether the marker in a new trial is predicting what you want to be predicting, you have to at least think about these issues. You may not want to deal with it because, ultimately, it is probably going to be difficult. In general, with unidentified problems including missing data problems, there's always these sorts of issues, which you need to deal with and that make things more difficult. But if you really want to fairly categorize the uncertainty and whatever sort of bias there might be, you can address it via shifts and priors on the extrapolation distribution. It turns out that in Dr Baker's meta-analytic framework for evaluating potential surrogates, you can use a Bayesian nonparametric approach as well. You might wonder why I'm harping on Bayesian nonparametrics, but if we can avoid making strong parametric assumptions and still draw similarly powerful conclusions, that would be good. It turns out if you use Bayesian nonparametrics, the nonparametric "power" penalty is not necessarily large. With Bayesian nonparametrics, you often don't lose that much power and you can potentially even get tighter intervals on parameters, depending on the specific model and data.

Dr Joffe's talk touched on causal effects with time-varying treatments. ${ }^{4}$ He first discussed global effects, meaning the effect of two treatments, either sequential treatments or two different treatments at different times; the key ideas are similar to a single treatment setting, both with principal stratification and with other causal identifiability approaches. But once you get to local approaches where you are holding one constant and varying the other, it gets much more difficult. There is going to be more to the extrapolation piece in those settings. In terms of the identifiability for the single setting, there will be similar issues, but it is going to be harder. But when it is harder, on some level, it is a little bit more interesting.

\section{Constantine Frangakis and Vivek Charu}

I will give a brief summary of my understanding of the essence of the three preceding articles and then I will discuss two issues in more depth.

First, Dr Taylor discussed the problem of using principal stratification as a way of evaluating the importance of a surrogate in predicting the effect of treatment. To address the identifiability issues, he presented the interesting idea of placing ordered assumptions in the correlations between the partially unobserved potential outcomes. He then used a gradation of strength in the assumptions on the order constraints, which allows one to see how the results change across that gradation. Dr Joffe discussed the literature on direct and indirect effects, as it gives insights into surrogacy. Of particular interest was the distinction between associational and causational measures of surrogacy. I think, however, that these two measures (goals) should somehow be related. In fact, we saw a possible such relation in the article by Dr Baker, in terms of the choice of the features of the potential outcomes that one believes are generalizable from one study to the other. More precisely, I believe that a connection between causation and prediction arises if it is a causal mechanism (as opposed to a noncausal one) that is more generalizable from one study to the other. Dr Joffe also discussed extensions to time-varying treatments. Finally, Dr Baker presented two additional 
ideas. In one, cross validation across trials was used to evaluate the errors of different ways of generalizing to a new trial. In the other, a method was used to collapse many baseline markers to a scalar, in order to better estimate heterogeneity of an effect.

There has been growing use of "principal stratification" for assessing surrogacy, but one issue needs more attention. Specifically, principal strata address the possibility that the manifestation of different surrogate values for different people can reflect partly unmeasured characteristics of a person. Therefore, it may not be wise to assume ignorability, for example, of a putative surrogate marker. However, if principal strata are used completely as confounders, then no variation of the putative marker is left to be interpreted as experimental variation. A suggestion to address this problem is to try and decompose the variation of principal strata into a "confounding" component, that is, one which needs to be conditioned on, and a residual component that can be used as an experimental, exposure factor. Figure 1 shows how this decomposition can be useful in a problem we had studied with Taki Shinohara (unpublished work). In that problem, a genetic factor had been found to be associated with uric acid across individuals, and we used that genetic factor as a "Mendelian randomizer" to examine whether uric acid affects the probability of gout. Figure 1 deals with an "index" patient (characterized by a set of covariates $x$ ) with a uric acid of $4.7 \mathrm{mg} / \mathrm{dL}$, and for whom we ask, if a treatment can decrease uric acid to 4 from 4.7, what would then be the expected probability of gout? To address this question, we matched that person, who has the control variant of the gene, to person $j$ who has the wild variant of the gene, so that they have similar values in two key variables: the predicted uric acid, say $\hat{U}\left(0 ; X_{j}\right)$, under the control genetic variant, for person $j$ is close to the value $\hat{U}(0 ; x)$ of the index patient; and the predicted uric acid, say $\hat{U}\left(1 ; X_{j}\right)$, under the wild genetic variant, for patient $j$ is close to the value $\hat{U}(1 ; x)$ of the index patient. (This matching is done partly based on the observed values of the surrogate and partly based on the predicted values because not all these values are observed for every person.)

With matches obtained based on closeness defined by a caliber, we conducted a local regression of the outcome-gout—on the uric acid. Figure 1 shows in color the regression lines for different choices of the caliber. What we observed from this approach is that as the caliber is allowed to shrink, the resulting regression lines start stabilizing, and, for small calibers, the lines start diverging again. This is interesting because, under certain conditions, it suggests that the stabilizing regression lines have conditioned on all the confounding aspects of the principal strata of uric acid as functions of the genetic factor.

The first topic of Dr Baker's presentation was the characterization of highly responsive patients based on multiple covariates. The motivating idea is that if one first summarizes the multiple covariates into a scalar score, then effect of heterogeneity on that scalar can be assessed empirically. ${ }^{5,6}$ The discussion below elaborates on a possible further development on this idea using the CitAD trial as an example. ${ }^{7} \mathrm{CitAD}$ was a randomized controlled trial designed to evaluate the efficacy of citalopram for reducing agitation in patients with Alzheimer's disease. The estimated overall treatment effect was a $13 \%$ increase in patients who improve substantially from baseline, when those assigned to citalopram are compared to those assigned to placebo. Because of possible adverse events associated with the treatment, a reasonable further goal for physicians might be to characterize and treat a 
subgroup of patients for whom the expected improvement is on average greater than, say, eff $_{\text {min }}=30 \%$.

For this goal, the existing approach would suggest the following. ${ }^{6}$ First, fit a parametric working model (which may not be correct): $\operatorname{pr}\left(Y_{i}=1 \mid X_{i}\right.$, Treat $\left.i, \beta\right)$ by the MLE $\beta$, where $X_{i}$ are covariates; Treat $_{i}$ is the treatment ( 1 if citalopram, 0 if placebo); and $Y_{i}$ is the indicator for substantial improvement from baseline. Then obtain an initial estimate of the effect $E\left(Y_{i}\right.$

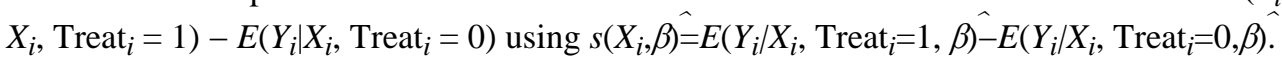
Subsequently, for every $s$, obtain empirical (nonparametric) estimates, say $\widehat{e f f}(\hat{\beta}, s)$, of

$$
\operatorname{eff}(\hat{\beta}, s):=E\left(Y_{i}(1)-Y_{i}(0) \mid X_{i}: s\left(X_{i}, \hat{\beta}\right) \geq s\right)
$$

and plot that against the percent of people with $s\left(X_{i}, \beta\right) \geq s$. For CitAD, this plot is shown in Figure 2. Finally, find the value of $s$, say $s^{*}\left(\hat{\beta}\right.$ eff $\left._{\text {min }}\right)$, that gives the largest proportion of patients for whom the effect $\widehat{e f f}(\hat{\beta}, s) \geq \operatorname{eff}_{m i n}$. This creates a treatment regime

$$
\operatorname{treat}\left(X_{i}, \hat{\beta}, \text { eff } f_{\min }\right)=\text { citalopram if } s\left(X_{i}, \hat{\beta}\right) \geq s^{*}\left(\hat{\beta}, \text { eff } f_{\min }\right)
$$

For example, Figure 2 shows that in order to have an average effect of at least $30 \%$, this approach finds that treatment can be given to at most $62 \%$ of the patients. ${ }^{8}$ While the above approach is useful, it gives rise to two problems as described below.

The first problem is that by finding the largest number with a large effect based on the above approach, we risk including patients with baseline covariate profiles for whom there is a negative treatment effect. The second problem is that because the working model is not necessarily correct, the MLE $\beta$ may not be the best estimate (even in large samples) to characterize a regime that would treat the largest number of people with large effect. To avoid the first problem, we consider the stratum of patients (by $x$ ) who would not be harmed by the treatment. This stratum can be estimated as

$$
\operatorname{not} \operatorname{harmed}\left(X_{i} ; \hat{\alpha}\right)=E\left(Y_{i} \mid X_{i}, \text { Treat }_{i}=1, \hat{\alpha}\right)-E\left(Y_{i} \mid X_{i}, \text { Treat }_{i}=0, \hat{\alpha}\right)>0
$$

where the model can be as before and $a$ is estimated semiparametrically as in Zhang et al. ${ }^{9}$

Now, a simple revision of the procedure outlined in equation (1) that addresses the above two problems is as follows: First, characterize the set of not-harmed patients, as above. Then, among these, consider the consequences if an arbitrary $\left(\beta, s^{\prime}\right)$ subset $\left\{s\left(X_{i}, \beta\right)>s^{\prime}\right\}$ of patients, where $s\left(X_{i}, \beta\right)$ is the effect based on the working model $s\left(X_{i}, \beta\right):=E\left(Y_{i} \mid X_{i}\right.$, Treat ${ }_{i}=$ $1, \beta)-E\left(Y_{i} \mid X_{i}\right.$, Treat $\left._{i}=0, \beta\right)$, receives treatment: a number of patients $N\left(\beta, s^{\prime}\right):=\#\left\{i: s\left(X_{i}\right.\right.$, $\beta) \geq s^{\prime}$ and not harmed $\left(X_{i}, a\right) \hat{\}}$ will have an average effect 


$$
\operatorname{eff}^{*}\left(\beta, s^{\prime}\right):=E\left\{Y_{i}(1)-Y_{i}(0) \mid X_{i}: s\left(X_{i}, \beta\right) \geq s^{\prime} \text { and not harmed }\left(X_{i}, \hat{\alpha}\right) .\right\}
$$

Suppose we wish to give treatment only if the effect in equation (2) is greater than the threshold eff $\min _{\text {m }}$. Then, for any particular $\beta$, we should identify the largest subset of patients for whom the average effect in equation (2) is larger than the minimal threshold eff min $_{\text {min }}$

$$
\text { \#largest- } \operatorname{subset}(\beta):=\max _{s^{\prime}} N\left(\beta, s^{\prime}\right)
$$

with average effect eff ${ }^{*}\left(\beta, s^{\prime}\right) \geq \operatorname{eff}_{\min }$.

Therefore, we should identify the largest subset of patients who can benefit from treatment by more than the minimal effect, as

$$
\text { \#largest- subset:= } \max _{\beta}\{\# \text { largest- } \operatorname{subset}(\beta)\} \text {. }
$$

By construction, this subset will be at least as large as the one characterized by equation (1), and it will be of interest to study how important that difference can be. Care should also be taken to avoid over-fitting while implementing this procedure.

\section{Debashis Ghosh}

Dr Baker had three "mini talks" on biomarkers with different uses; Dr Taylor focused on looking at surrogacy in a causal modeling framework; and then Dr Joffe talked about a more conceptual framework that mimics medical decision making and applies causal inference in that set up. This is not the order in which you heard the talks, but the way I've ordered these talks is actually the way one thinks about association versus causation. The majority of $\mathrm{Dr}$ Baker's presentation was focused on inference based on using association measures, and he talked about extrapolation. Dr Taylor then worked in the potential outcome framework using principal stratification and then Dr Joffe offered even more complicated causal modeling ideas. One theme of this session that I would like to hear the speakers say more about is the inherent tension when you do surrogacy modeling, thinking about association versus causality or causal modeling or causal effects. By "association" what I am referring to is a quantity that you can compute and estimate from the observed data. Dr Daniels alluded to this in his discussion. It is easy to check whether the model is holding true or not. The problem is that in a lot of situations what we care about scientifically is what I am calling causal estimands, and those are going to require a lot more assumptions. Dr Taylor gave a nice example in his application with 14 parameters of which only 10 could actually be identified from the observed data. That is the inherent tension: do you want to base inference only on the observed data or try to do causal modeling, which requires more than the observed data. There are lots of causal modeling frameworks that you can use-Dr Joffe called them "languages for causal inference." Dr Taylor worked mostly with the potential 
outcomes framework. Dr Joffe, in his talk, showed you a lot of graphs (which Judea Pearl ${ }^{10}$ calls causal diagrams).

Fundamentally, this issue of causality becomes more of a study design problem; surrogacy inherently relates to something that happens after the treatment gets assigned. If you want to assess how a post-treatment event affects your true outcome, you are talking about an embedded observational study. Even if your initial trial has been randomized, when you start looking at post-treatment events and trying to figure out what their effects are in your experiment, you are talking about a question that is basically an observational data analysis question, not a randomized trial question. You make a lot of assumptions when you do causal modeling. People allude to using sensitivity analysis, but it seems like another good thing to do is assume you have the wrong model to start with. In other words, do your causal modeling based on some true model and then ask, since I used the wrong model, how bad is my final answer; how sensitive are my results to the wrong model choice? Also, multiple assumptions are generally needed for defining causal estimands. One can also consider simultaneous sets of assumptions, exploring them in sensitivity analyses, rather than fixing all but one parameter and doing sensitivity analysis just on that; looking at multiple parameters simultaneously should give you more information about the sensitivity results.

My next point is a historical throwback. People used to think a lot about model misspecification in the 1960s and 1970s, with Kullback-Leibler divergence and least false parameter minimizers; that time period was when the whole idea of sandwich variance estimators started. Let's say I have these assumptions I need for valid causal inference, but I don't know them to be true. Is it possible to think of constructing analogies to sandwich variance estimators, which might have some robustness built in against starting with the wrong assumptions? I think Dr Daniels alluded to this in a more nonparametric way when he talked about using nonparametric priors. I think a lot about combining approaches. If you think of association based on the observed data on one end of a spectrum and causal modeling on the other end, is there a hybrid approach somewhere in between? One example is work by Booil Jo et al. ${ }^{11}$ on what she calls reference stratification.

I have a few comments about surrogacy and biomarkers. One question is whether the surrogate endpoint we care about is something that we can intervene on- whether the surrogate is manipulable or not. I think this was Dr Joffe's point about whether you have a proxy surrogate versus a causal intermediate. Another issue is timing of the measurements relative to the true endpoint. Drs Baker and Taylor both brought this up; one reason we look for surrogate endpoints is to see whether we can find an endpoint that occurs much earlier so that we can stop the trial sooner. Basically, the potential outcomes framework does not allow for manipulability. What it does is say is that I have my surrogate outcomes under my hypothetical treatment regimes. I am going to treat them like baseline covariates. Since you condition on those, you are basically treating them as fixed. The causal diagrams that Pearl talks about do allow for manipulability of surrogates. Robins and Greenland ${ }^{12}$ in 1992, in causal diagrams, manipulate the surrogate by the do-operator calculus of Pearl. ${ }^{10}$ This means you basically set the surrogate to some value and see how it affects the outcome. The question again is whether this is a complementary causal modeling framework and can you think about whether the surrogate is manipulable. The standard statistical answer is "it 
depends." It is going to depend on the context you are working in, as well as how much biological knowledge you have about the endpoint. Timing of the surrogate-this goes back to one of Dr Baker's points: if the goal is to have shorter trials, you basically want surrogate endpoints to have greater predictive power at earlier time points that can predict true endpoints at later time points. Dr Taylor presented a causal estimand in his talk; my thought was perhaps you could allow for the response variable to depend on time and look at the potential outcomes at time $\mathrm{T} 1$ given surrogate measured at time $\mathrm{T} 0$. The hope would be that $\mathrm{T} 0$ would be a lot smaller than $\mathrm{T} 1$ and you would have a strong surrogate in the sense that he described so that my study could be more efficient. I am not sure if that is feasible or not.

Other causal modeling frameworks exist; an interesting one is an extension of the framework that Pearl outlines, which has been well developed in economics. In that framework by definition you are not going to be able to separate out post-treatment selection effects of the subjects from the ability of the surrogate to predict the true endpoint. The implication with this framework, then, is that trying to find good surrogate endpoints will take as long as doing a study with the true endpoint, which defeats the purpose of doing the study.

The other thing I want to touch on is extrapolation. If you assume that the probability of the treatment effect in the historical trials was the same as the probability of the treatment effect in the new trial, then the relationship between the true endpoint and surrogate from the historical trial was the same. This idea is something that has been formalized recently by Bareinboim and Pearl ${ }^{13-18}$ in a series of articles; they call it transportability or metatransportability. Pearl has developed some new graphs, called selection diagrams, to illustrate this concept. I have used this approach in one study. I have the results, and I now want to move to a different study population. Pearl describes the conditions you need to guarantee that your results from the study can be transported to the next study. It turns out that if you want to transport the causal effect to the next study, you would have to re-weight the analysis to your new population based on the distribution of the entry criteria for the study. In epidemiology, we have standardization of rates, so this kind of graph would correspond to that kind of re-standardization. This approach is useful for thinking about whether or not results are generalizable or whether Dr Baker's extrapolation would hold.

To wrap up, causal inference allows you to separate the science from the data you have collected. The key point in all these talks relates to how much extrapolation or prediction or imputation of the missing data can be done; and fundamentally, there are no great ways to check this because it is inherently nonidentifiable. One thing we recommend people do in an observational study is collect information that might be related to your post-treatment events of interest; if you do that, then potentially you might have certain conditional independence assumptions holding that you need for valid causal inference.

\section{Stuart Baker}

I want to make two points. First, a theme of the panelists was a comparison of causal assumptions and associational assumptions. My view is to treat this empirically. I fit the principal stratification model and I fit the linear random effects model and I compare them using a leave-one-out approach with historical trials. Second, we have seen some 
mathematically clever approaches with surrogate endpoints. But it is important to not lose sight of the extrapolation to a new trial. The biology can be so complex that what holds in historical trials may not apply to a new trial.

\section{William L Mietlowski}

I am interested in randomized phase II trials in oncology, where the phase III trial is based on overall survival (OS), so it is an intermediate endpoint problem. Hui Gan, an oncologist, in a 2013 personal communication indicated that of 120 randomized phase III oncology trials with an OS primary endpoint, about $30 \%$ had a statistically significant treatment effect. If one has the raw datasets from randomized phase III trials with known overall survival outcomes, one can simulate randomized phase II trials to come up with estimates of sensitivity, specificity, positive predictive value, and negative predictive value for various intermediate endpoints. The positive predictive value of current randomized phase II trials may be unsatisfactory if the success rate in phase III is only $30 \%$.

\section{Elizabeth Kumm}

I am a former student of Dr Ghosh, and I have a point about wanting to end a study early based on a really good surrogate. There are cases, especially in oncology, when you may have a good marker but don't necessarily need to end the study earlier on the basis of the marker. I am thinking about circulating tumor cells, which are easier to assess than cells obtained from an invasive biopsy. The marker might not allow you to end the study earlier, but you can still get a benefit from that kind of information.

\section{Debashis Ghosh}

Elizabeth, your comment gets to the point of having to think carefully about the role of the biomarker. It might not be to replace the endpoint of interest. It might just be to better manage the process or do better stratification.

\section{Marshall Joffe}

I wanted to make a few comments, mostly in response to some of Dr Ghosh's observations. The first issue has to do with certain problems when conducting sensitivity analyses in highdimensional settings. There is an issue of interpretability if there are many sensitivity parameters. If there is one sensitivity parameter, then it is fairly easy to do a sensitivity analysis; you vary that sensitivity parameter over some plausible range and you look at what happens - it is easy enough to do. If you have five sensitivity parameters, then it's a lot harder to be guided. You can vary one at a time, which will only cover a tiny proportion of the parameter space that you are interested in. You can look at bounds, which often will be so broad as to give you nothing that's useful. A few of the discussants, and also Dr Taylor, take a Bayesian approach, which in some ways is a nicer approach because then you can at least get something a little bit informative. As is typical in a Bayesian setting, the quality of your inference is going to depend to some extent on the quality of your priors, which you can then vary over some plausible range. 
The second issue that Dr Ghosh brought up was model misspecification. To some extent, I think what you are interested in estimating in the presence of misspecification may be more interesting than variances you get from a sandwich or a robust variance estimator. I think others may have dealt with this; Gruber and Mark Van der Laan ${ }^{19}$ and his group have thought deeply about this issue. They like to define things nonparametrically and then project what you do parametrically upon the nonparametric estimands and see how well they are estimating the quantities that you've defined, in a way that doesn't depend on your parametric models.

A third issue has to do with the principal stratification approach and the manipulability of intermediate variables. My understanding of the principal stratification approach is that it is essentially agnostic about the manipulability of the intermediate variables or the surrogates. In other words, it doesn't assume that they are or are not manipulable.

The last issue has to do with the transportability that has been discussed by Pearl and Bareinboim ${ }^{13}$ and others. One of the issues I have is that their selection diagrams are largely dealing with perfect surrogates in the surrogate outcome problem. My concern with that, as it is with the Prentice ${ }^{20}$ criterion (which people have relaxed), is that perfect surrogates are essentially impossible to find and they create an ideal situation that no surrogate is ever going to be able to satisfy. Somebody is going to have to come up with some way of dealing with imperfect surrogates or imperfect transportability. What has been done is just a start, and it needs to be made more useful in some fashion.

Finally, in terms of the comments about sensitivity and specificity, I don't like that it involves dichotomizing what are essentially nondichotomous results. To some extent the meta-analytic paradigm, for example, that Dr Daniels was instrumental in developing, doesn't impose such a requirement.

\section{Jeremy Taylor}

Dr Daniels talked about checking goodness of fit. For the strategy you suggested, you model the observed data, which you can do, and then you have to deal with the unobservable part, or the nonidentified part. You can make some assumptions to get rid of some parameters; then you are left with other parameters which you can regard as sensitivity parameters. These can be varied to see how they affect the results. In my particular case, without making assumptions, there would be four sensitivity parameters. If you weren't willing to make any assumptions, you've got to figure out how to do a sensitivity analysis in a meaningful way by varying four parameters and try to understand what it tells you, so it becomes a little tricky.

\section{Michael Daniels}

In your setting, given how you framed it, the parameter space of the sensitivity parameters is a nice-bounded space, so it makes it much more manageable to do sensitivity analysis. The problem with sensitivity analysis that Dr Joffe was just talking about can be dealt with easily if you make parametric assumptions. But the thing is, even with priors, if you have a 
compact space, it's much easier to think about putting priors on parameters, too. Outside of bounded spaces, it becomes more tricky to assess sensitivity.

\section{Jeremy Taylor}

I presented a Bayesian approach, which requires prior distributions. If you use very flat noninformative priors you haven't really solved your problem. You might be able to do some estimation because you can get algorithms to run to give you estimates.

Nonidentifiability means two sets of parameters give you the same likelihood, so if you put no information in the priors you're not going to gain much. You really have to use informative priors to some degree. The other comment I have about this is, if you do Bayesian analysis with not super informative priors and large samples for a fully identified model and you look at credible intervals, then the coverage rate of credible intervals is approximately right from a frequentist perspective. If you have nonidentified models and you use Bayesian priors, then you don't necessarily get good coverage rates even with large sample sizes. You can run into trouble. The fortunate thing is the intervals tend to be conservative. If you are presenting some uncertainty interval from a nonidentified model with somewhat informative priors, then coverage rates which you hope to be $95 \%$ may actually be $97 \%$ or $99 \%$; that's been our experience.

People talked about generalizability from one trial to the next. Suppose you can somehow establish that a surrogate is good in this trial, then can you hope for it to be good in the next trial? Dr Joffe made comments about this. What might be generalizable are mechanistic aspects. You don't expect marginal distributions or treatment effects to be the same from one trial to the next, but may be the causal mechanistic aspects are. The causal ways of thinking about the problem allow you to focus on specific aspects that might be related to the underlying mechanisms. If those were fairly consistent from one trial to the next, even with different drugs, you might be more willing to believe that, in the next trial, those aspects of the distribution will be preserved. That's how you may be able to generalize to new trials.

Regarding timing of the surrogate and the true endpoint- I like to think of a spectrum; there's an intervention at one end and there is a true endpoint at the other end. There is a time frame between them and you can think, where on that time frame does the surrogate lie? It might be near the true endpoint-for example, if overall survival is the real endpoint, then something occurring close to the time of death would be a potential surrogate. An example at the other end of the scale: you have a therapy that is trying to change the immune system. Then, a potential surrogate could be some measure of the immune system, which you are going to have soon after start of therapy. This would be at the other end of the timescale. It is probably going to be harder to find good surrogates very near the time of treatment that are going to generalize to other trials. That is because the choice of surrogate would typically be rather specific to the treatment, and the causal relationship between the surrogate and the true outcome may not be strong. But the causal relationship between near death and death is probably going to be preserved. So, my opinion is that we are more likely to have success in establishing surrogates that are closer to the true endpoint. 
Dr Ghosh made a comment about the amount of data that is needed, and that if you have data from the full trial anyway what is the point in trying to establish the usefulness of a surrogate. He was talking about the treatment effects on the true endpoint at time $\mathrm{T} 1$ given the effect on the surrogate at time T0. One question is, can you shorten the trial? To do the estimation you need good follow-up data on some people, not necessarily everyone. If you are going to analyze data from a trial, people enroll over a time span of 4 years, say, and then there is a certain amount of follow-up. The first enrollee has a long data history and the last enrollee has a short data history, so you have some information out to the longest follow-up time. You don't necessarily need everyone to have full follow-up. You can potentially extrapolate for an individual out to that range where you've got some data on other people and still stay within the bounds of the data, conditional on the models that you are specifying.

There was a comment about randomized phase II trials in oncology and that their results haven't been that successful at predicting what is going to work in phase III trials. I think the point was if we could have all the data from those randomized trials maybe we could analyze it and learn about the causal relationships within those trials, and with that original raw data available, one could make better choices about what might work in phase III, rather than just relying on significance tests from typically too small phase II trials. Phase II trials in oncology typically aren't big enough to get much confirmatory evidence. Rather than only looking at whether a significance level is attained or not in a phase II study and assessing whether that predicts the phase III result, it might be useful to try to learn from the data about the causal mechanisms that might be more informative about whether to go on to phase III.

\section{Songbai Wang}

Traditionally, I think a lot of people use the Prentice criteria to evaluate a biomarker to see if that can be classified as a surrogate endpoint and now we have been discussing a new methodology. Is there any connection between the Prentice criteria and other methods such as principal strata?

\section{Jeremy Taylor}

Yes, there is. With the Prentice criteria, you model the outcome, condition on the surrogate, and the treatment, hope that the coefficient associated with treatment is zero and then you would say that conditional on the surrogate the treatment doesn't matter. That's essentially the main Prentice criterion. It is quite connected to the graphical causal models that Judea Pearl and others have talked about. One of the graphs I presented was a graphical model, and the Prentice criterion is related to these types of models. If you are willing to make the assumption of no unmeasured confounders, then the Prentice criterion can have a causal interpretation. In causal inference, other covariates are commonly added to the regression models to make the assumption of no unmeasured confounders more plausible. The Prentice approach doesn't typically have covariates on the right-hand side of the model, but if you start adding those in the models that you fit, then you might be more willing to make the type of unverifiable assumptions you need to make for the Prentice criterion to be valid from 
a causal perspective. So, the Prentice criterion does have some connection to the graphical model causal framework, not quite so much to the principal stratification framework.

\section{Constantine Frangakis}

I would like to add a little bit to the discussion that Dr Joffe opened up, about sensitivity. Would it be possible to address sensitivity to adding a possible covariate that you haven't measured by subtracting a variable that you have measured? For example, if you have 10 measurements relating to the baseline health of an individual, and you think that you haven't measured them quite accurately, then you could possibly think of an analysis that first uses all of them and then starts tapering off the degree to which you are using these measurements. If you start tapering them off and you see that the result changes drastically in the neighborhood of where your original result was, then you would be worried, because it would suggest that the result would have continued to change if you had added even more detailed information than the one you have. If, however, as you taper off the use of these measurements, by some way, for example, principal components, you see that the results do not change, then that would be more consistent with the hypothesis that you have measured that dimension of health well enough. Of course, there cannot be proof of that because there can always be something unmeasured that is affecting the results, but it is qualitatively different to see the results changing dramatically near your full answer versus not changing. I think that would raise flags.

\section{Debashis Ghosh}

I thought that what Dr Frangakis was describing was in a way like the covariate version of the empirical "leave one out" that Dr Baker was talking about, but robustness of answers that way would be important to check for.

\section{Stuart Baker}

The Prentice criterion was originally formulated for hypothesis testing. Principal stratification was not, but I developed a modified version applicable for hypothesis testing.

\section{Herb Weisberg}

I think what we have talked about today is very helpful in terms of the narrow problem of how you can use a surrogate endpoint to say something about a specific endpoint of interest in a particular trial, but it seems to me that there's a much broader potential value for looking at surrogate endpoints because they do tell you something about the biological processes that are going on. I don't know if much thought has been given to the assembly across different fields of investigation of different sets of responses that you are getting in clinical trials and capturing that information to see whether the medical researchers can put that together in some meaningful pattern and perhaps learn something that you are not getting from focus on any one individual study. 


\section{Michael Daniels}

Are you thinking about something different from just a meta-analytic framework; or are you getting at something more than that?

\section{Herb Weisberg}

I am talking about this more as an area of research that often doesn't get addressed. I am talking about looking at it from the biological standpoint, not in terms of trying to put it all together in some meta-analytic way, but in terms of looking for what's responding and what isn't and seeing whether one can find any meaningful relationship to causal mechanisms that may be known and that might help shape research going forward. It's sort of about research in general, not about testing for something specifically in a confirmatory way; it's more in an exploratory vein.

\section{Jeremy Taylor}

There are lots of intermediate variables measured throughout trials. So yes, I would agree. If you can basically understand the whole system of how those intermediate variables relate to the treatment and how they relate out to the outcome then that would be very helpful. It changes the focus from a specific surrogate outcome to understanding the whole system.

\section{Chen $\mathrm{Hu}$}

I am just curious if the panel has thoughts about whether there are ways to improve the efficiency of clinical development by prospectively evaluating surrogacy at the design stage. For example, in some settings, progression free survival (PFS) is a putative surrogate for overall survival. How would you prospectively evaluate the putative surrogacy of PFS for overall survival in a phase III trial design?

\section{Jeremy Taylor}

You can simulate data where you have both surrogate and the true outcome measured and see what you can learn from that. This raises a different issue. Surrogacy is all about replacing the true endpoint with a surrogate endpoint. Another approach involves auxiliary variables where you focus on the actual endpoint, but you use the information on the surrogate outcome and its association with the true endpoint to help improve the efficiency of a trial. That's not replacement, but that's utilizing information. So, in principle, you can utilize the large number of intermediate variables that typically get measured along the way in a clinical trial, but it requires you to build models and to have enough data to be secure about those models.

\section{Chen $\mathrm{Hu}$}

There has been a lot of development of methods for surrogacy evaluation, so I wonder, for at least phase III trial designs, if you still use some sort of conventional approach with, say, overall survival as a primary endpoint, can these new developments be used prospectively in the trial design phase, so that we can improve our decision-making process along the way? 
Or by the end of the study can we make a stronger statement than just doing some exploratory analysis?

\section{Stuart Baker}

In the future, you might not know the surrogate endpoint you want to study, but if you had some stored specimens, you might have some more options for selecting a future surrogate endpoint.

\section{Jeremy Taylor}

In a particular setting, you can imagine interim analyses that are based on some composite of the true endpoint and the surrogate in some way and deciding to stop early based on that composite. This would be a design phase aspect, so you don't just rely on the true endpoint at earlier times. You rely on the surrogate at earlier times to help you make decisions as you go through the trial.

\section{Acknowledgments}

AbbVie, Inc., and the Center for Clinical Epidemiology and Biostatistics at the University of Pennsylvania provided funding, scientific, or logistical support for the conference. The American Statistical Association and the Society for Clinical Trials provided in-kind support for the conference.

Funding

Funding for this conference was made possible (in part) by AbbVie, Inc. (205666). The views expressed in written conference materials or publications and by speakers and moderators do not necessarily reflect the official policies of the Department of Health and Human Services; nor does mention by trade names, commercial practices, or organizations imply endorsement by the US Government.

\section{References}

1. Taylor JM, Conlon AS, Elliott MR. Surrogate assessment using principal stratification. Clin Trials. 2015; 12:317-322. [PubMed: 25490988]

2. Baker SG, Kramer BS. Evaluating surrogate endpoints, prognostic markers, and predictive markers: some simple themes. Clin Trials. 2015; 12:299-308. [PubMed: 25385934]

3. Ellenberg SS. Surrogate endpoints. Br J Cancer. 1993; 68:457-459. [PubMed: 8353034]

4. Hsu JY, Kennedy EH, Roy JA, et al. Surrogate markers for time-varying treatments and outcomes. Clin Trials. 2015; 12:309-316. [PubMed: 25948621]

5. Byar D, Corle DK. Selecting optimal treatment in clinical trials using covariate information. J Chronic Dis. 1977; 30:445-459. [PubMed: 885985]

6. Cai T, Tian L, Wong PH, et al. Analysis of randomized comparative clinical trial data for personalized treatment selections. Biostat. 2011; 12:270-282.

7. Porsteinsson AP, Drye LT, Pollock BG, et al. Effect of citalopram on agitation in Alzheimer disease: the CitAD randomized clinical trial. JAMA. 2014; 311:682-691. [PubMed: 24549548]

8. Rein, L. Master of Science Thesis. Johns Hopkins University; Baltimore, MD: 2014. Assessing treatment effect heterogeneity in the Citalopram for Agitation in Alzheimer's Disease clinical trial: a subgroup analysis to guide personalized treatment selections.

9. Zhang B, Tsiatis AA, Laber E, et al. A robust method for estimating optimal treatment regimes. Biometrics. 2012; 68:1010-1018. [PubMed: 22550953]

10. Pearl J. Causal diagrams for empirical research. Biometrika. 1995; 82:669-710.

11. Jo B, Wang CP, Ialongo NS. Using latent outcome trajectory classes in causal inference. Stat Interface. 2009; 2:403-412. [PubMed: 20445809] 
12. Robins JM, Greenland S. Identifiability and exchangeability for direct and indirect effects. Epidemiology. 1992; 3:143-155. [PubMed: 1576220]

13. Pearl, J.; Bareinboim, E. External validity and transportability: A formal approach. 2011 JSM Proceedings; Miami Beach FL. July 30-August 4, 2011; p. 157-171.

14. Pearl, J.; Bareinboim, E. Transportability of causal and statistical relations: a formal approach. In: Burgard, W.; Roth, D., editors. Proceedings of the twenty-fifth national conference on artificial intelligence. Menlo Park, CA: AAAI Press; 2011. p. 247-254.

15. Bareinboim, E.; Pearl, J. Transportability of causal effects: completeness results. In: Hoffmann, J.; Selman, B., editors. Proceedings of the twenty-sixth national conference on artificial intelligence. Menlo Park, CA: AAAI Press; 2012. p. 698-704.

16. Bareinboim E, Pearl J. A general algorithm for deciding transportability of experimental results. J Causal Inference. 2013; 1:107-134.

17. Bareinboim, E.; Pearl, J. Causal transportability with limited experiments. In: DesJardins, M.; Littman, M., editors. Proceedings of the twenty-seventh national conference on artificial intelligence. Menlo Park, CA: AAAI Press; 2013. p. 95-101.

18. Bareinboim, E.; Pearl, J. Transportability from multiple environments with limited experiments: completeness results. In: Welling, M.; Ghahramani, Z.; Cortes, C., et al., editors. Advances of neural information processing 27; NIPS Proceedings; La Jolla, CA: NIPS; 2014. p. 280-288.

19. Gruber S, Van der Laan MJ. Consistent causal effect estimation under dual misspecification and implications for confounder selection procedures. Stat Methods Med Res. Epub ahead of print 23 February 2012. 10.1177/0962280212437451

20. Prentice RL. Surrogate endpoints in clinical trials: definition and operational criteria. Stat Med. 1989; 8:431-440. [PubMed: 2727467] 


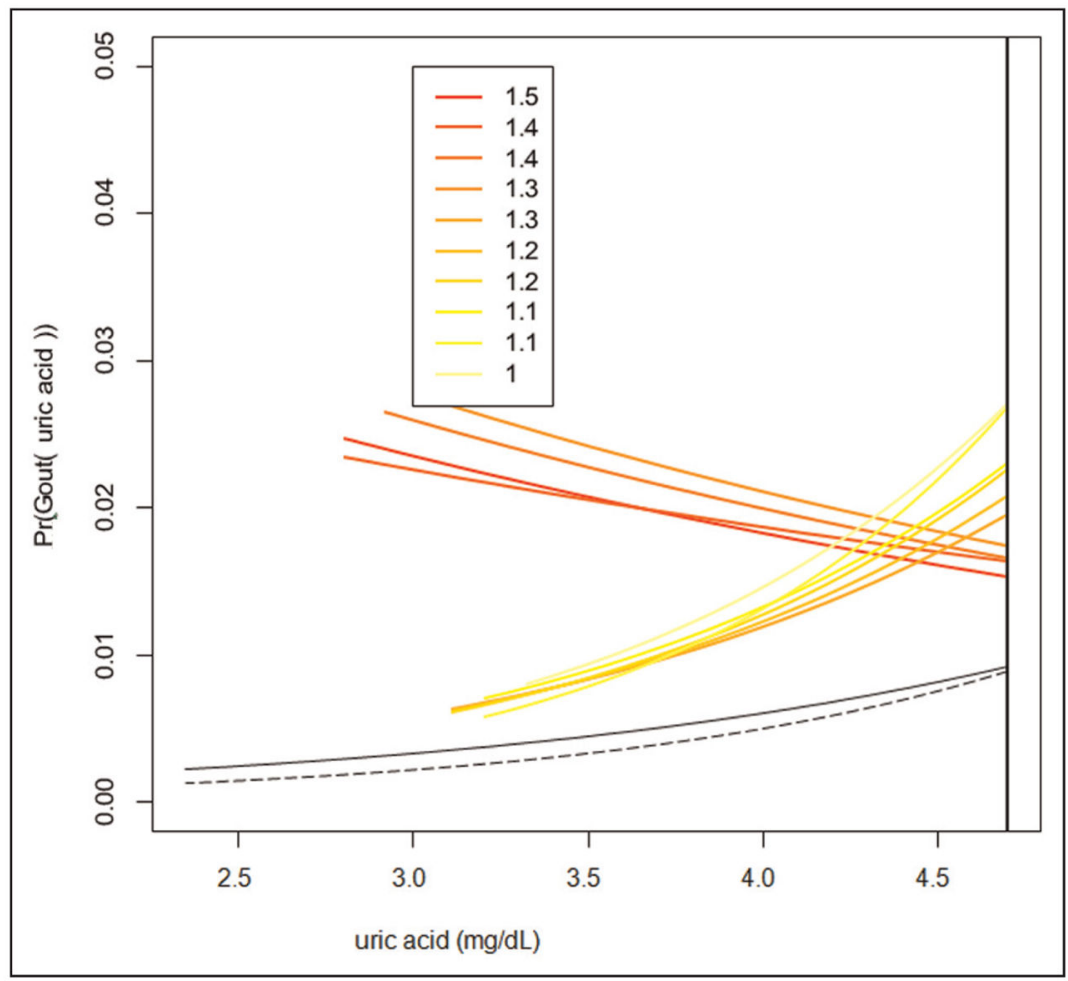

Figure 1.

Probability of gout as a function of uric acid. The black line here is the regression of gout on uric acid with no attention to genetic information. Color lines are from regressions with different decomposition of the uric acid variation into genetic and nongenetic components (see text). 


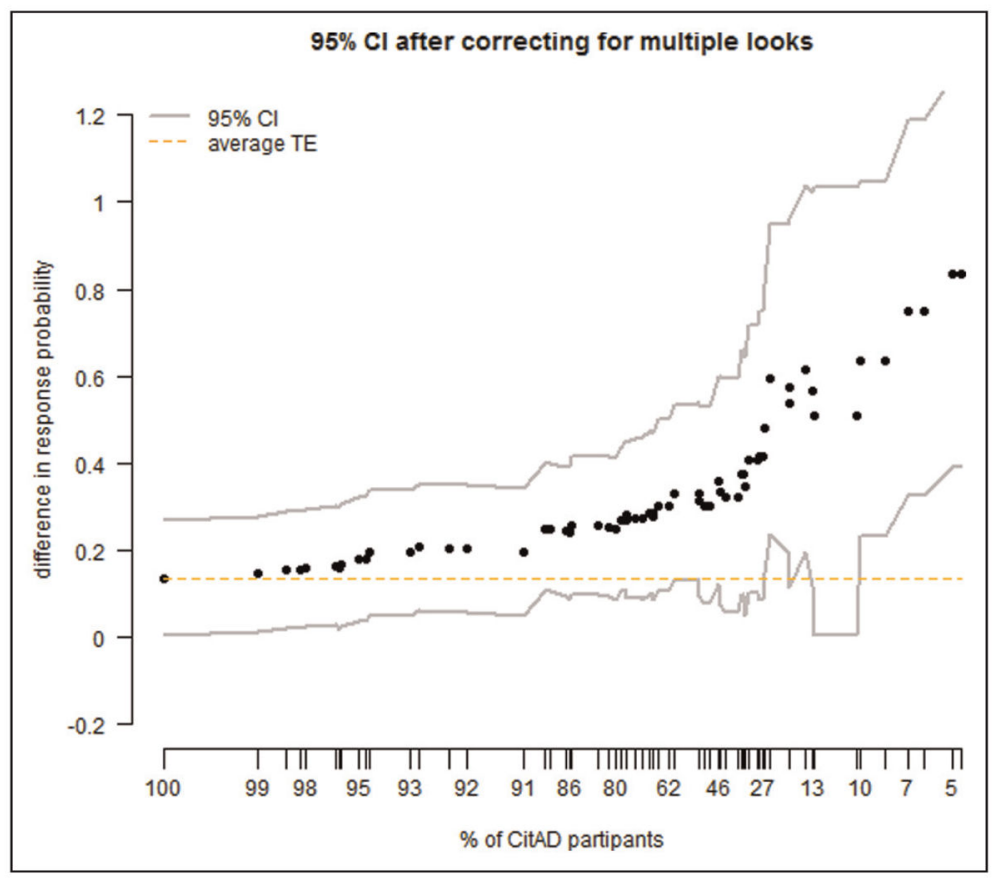

Figure 2.

Empirical estimates of $E\left\{Y_{i}(1)-Y_{i}(0) \mid X_{i}: s\left(X_{i}, \hat{\beta}\right) \geq s\right\}$ plotted against the percent of patients with $s\left(X_{i}, \beta\right) \geq s$ (for each $s$ ) in the CitAD trial. 